\title{
First Direct Observation of Biological Molecules in Liquid by Environmental Phase Contrast Transmission Electron Microscopy
}

\author{
Yuhri Inayoshi*, Hiroki Minoda* \\ *Department of Applied Physics, Tokyo University of Agriculture and Technology, Japan,
}

Structural investigation of protein motion is one of the important ways to understand cell motility. While direct observation of protein motion by TEM is the most direct way for this aim, there are two large requirements. One is to keep specimen in water and the other is to get high contrast of specimens. We could fulfill the former one by using environmental TEM (E-TEM) and the latter one by using phase contrast TEM (P-TEM).

E-TEM permits in-situ observation during chemical reactions under the various conditions such as in aqueous solution $[1,2]$. E-TEM used in the present study is a window type and windows for electron pass are on the top and the bottom of an environmental cell (EC) (fig. 1(a)). The EC is connected with a gas flow system as seen in Fig. 1(b) to circulate ambient gases around experimental solution.

P-TEM permits direct observation of biological molecules without any stain [3,4]. Using a Zrnike type phase plate which gives a phase shift of $-\pi / 2$ to scattered electron waves (fig. 1), nearly full potential distributions of biological molecules can be visualized. In this paper a first observation of biological molecules without stain in an aqueous solution by using the combination method between E-TEM and P-TEM will be presented.

For a comparison of images of the biological specimen in vacuum and the aqueous solution, the specimens in dry condition and in water are presented in figure 2. Figure 2 (a) and (b) showed myosin filament without stain in the dry condition. Figs. 2(a) and (b) are an amplitude image and a phase image, respectively. In (a), only dark images correspond to gold particles with $20 \mathrm{~nm}$ in diameter are seen in (a). They were attached to myosin heads as position markers. In addition to the gold particles the myosin heads with elliptic shape indicated by black arrowheads are seen in (b). Gold particles in (b) seen to be larger than the real size caused by a halo effect according to a finite size of a central hole of the phase plate. Figs. 2 (c) and (d) showed an amplitude image and a phase image of the myosin filaments without stain in aqueous solutions. A thickness of water solution was estimated to be about $100 \mathrm{~nm}$. There is no contrast in (c) but thin filaments and myosin heads are visible in (d). Though coherence of the electron wave is lower due to the effects of the water solution around the specimens, contrast enhancement by the phase plate was evident. In the present paper the effects of inelastic scattering on the image contrast will be also discussed.

\section{References}

[1] Fukami, A. et al., Microstructure of Fine-Grained Sediments from Mud to Shal, eds Bennett, R.H., Bryant, W.P. \& Hubert, M.H., 1991

[2] Sugi, H. et al., Proc. Natl. Acad. Sci. USA 105 (2008) 17396-17401

[3] K. Nagayama, J. Phys. Sci. Jpn. 69 (1999) 811-822.

[4] R. Danev and K. Nagayama, J. Phys. Sci. Jpn. 70 (2001) 696-702.

[5] We are thank to Mr. Arai in Tera Base Co. and Prof. Nagayama in National Institute of Physiological Science. 
(a)

(b)

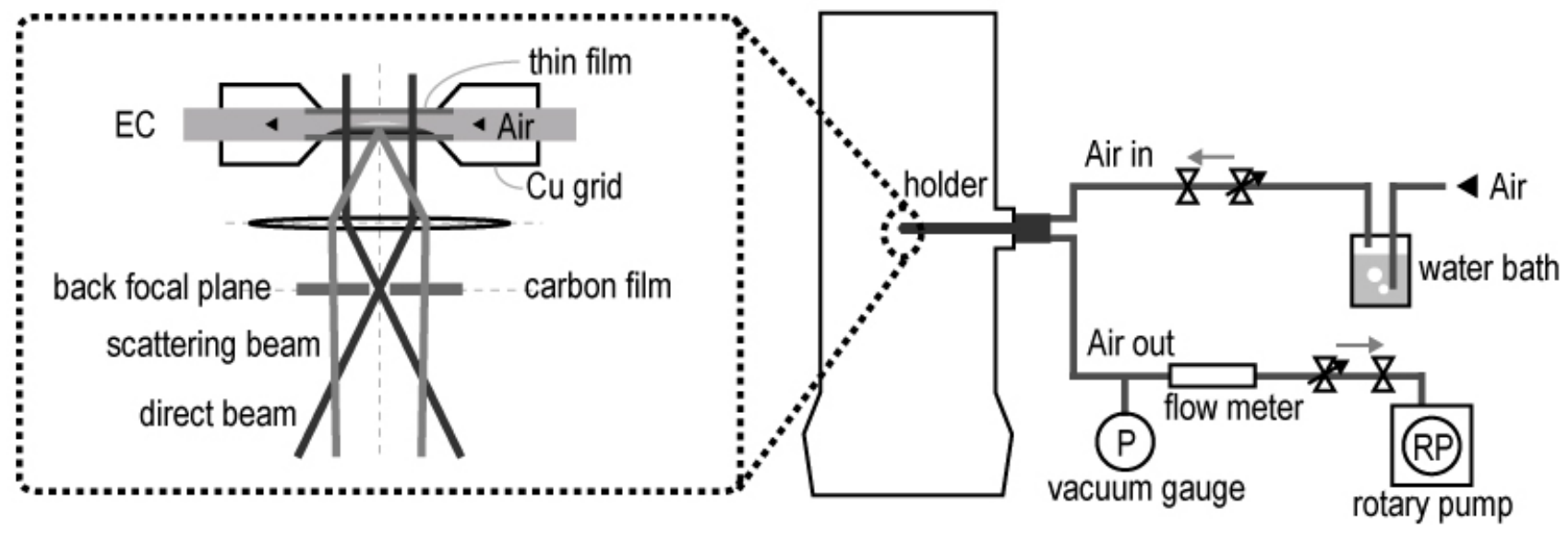

Figure 1

Schematic drawings of a ray diagram of the E-TEM P-TEM combination method in (a) and the gas flow system in (b). The Zernike phase plate of carbon thin film with a $1 \mu \mathrm{m}$ diameter hole at the center was placed at the back focal plane of the objective lens. Lateral position of the phase plate was adjusted so as to only unscattered electrons can pass thorough the central hole of the phase plate. Windows of a grid are covered with a carbon amorphous thin film with about $10 \mathrm{~nm}$ thickness. Specific gases or air can be flowed around the specimens. Pressure around the experimental solution can be controlled by changing a gas flow rate by monitoring vacuum gauges and gas flow meters.
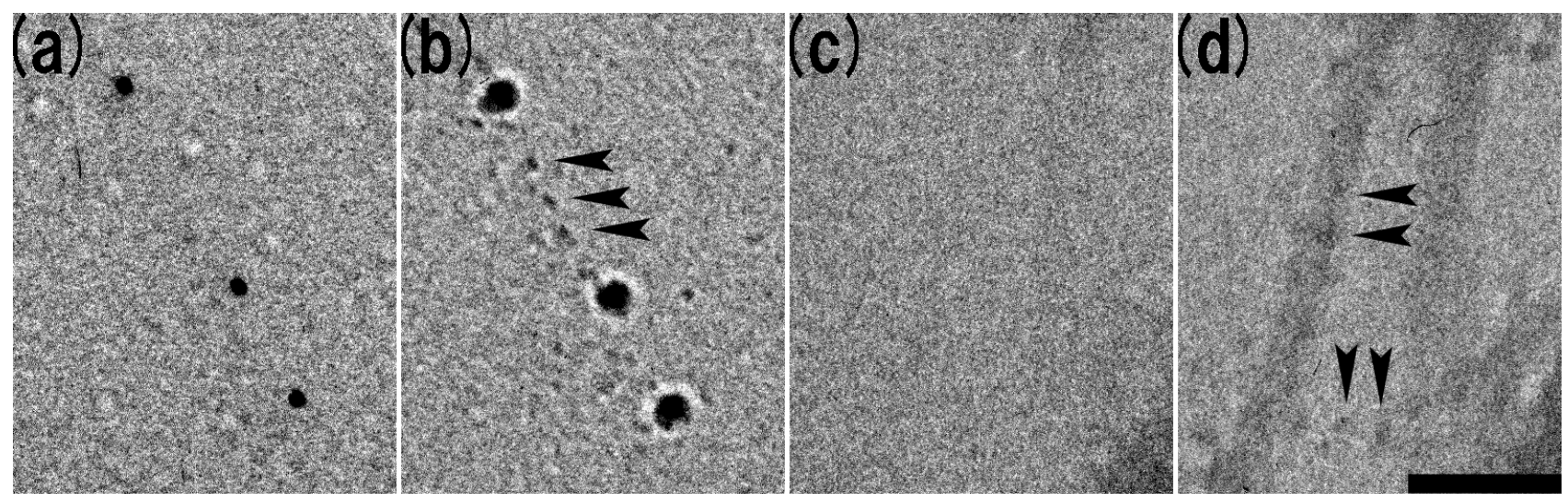

Figure 2

Figs.2 (a) and (b) show conventional-TEM and P-TEM image in vacuum, respectively. Figs.2 (c) and (d) show conventional-TEM and P-TEM image in aqueous solutions. An arrowhead indicates a myosin head. A scale bar corresponds to $100 \mathrm{~nm}$. Contrast enhancement of the images by P-TEM was evident for the specimens in vacuum and in water solution. The phase image of the specimens in vacuum is much sharper than that in water solution. 